\title{
Los sismos de septiembre y la salud en México
}

\author{
Julio Frenk, * Miguel A. González* y Jaime Sepúlveda**
}

El artículo examina los efectos que causaron los sismos sobre ia salud de la población y sobre ia infraestructura de los servicios de salud en México, así como su incidencia sobre el desarrollo de la reforma planteada para ese sector por la actual administración. Para ello se analizan las características generales de dicha reforma, los obstáculos que enfrentaba y los problemas que surgieron a raíz de los sismos. Se concluye que a pesar de que el sector salud fue el más afectado por la pérdida - sin precedentes en la historia nacional y mundial - de profesionales, instalaciones y equipo, la catástrofe permitió profundizar la reforma del sector y coadyuvar a una mejor distribución de los recursos para la atención médica.

Los sismos de septiembre de 1985 tuvieron un gran impacto sobre la estructura de ios servicios de salud en México. Más de $10 \%$ de las vidas que cobró el desastre se perdieron en los hospitales derruidos, mientras que en un solo día dejó de existir una cuarta parte del total de la capacidad hospitalaria del sector público en la zona metropolitana. Estos hechos se insertan a su vez en el contexto de una profunda crisis económica, a la cual el sector salud intentaba hacer frente por medio de una reforma estructural sin precedentes. Consideramos pertinente entonces examinor los efectos que han tenido y tendrán los sismos de septiembre sobre el desarrollo de la reforma al sistema de salud en México. Abordaremos este problema analizando las características generales de la reforma propuesta a principios deI presente sexenio, los problemas que ya de por sí enfrentaba y los daños que los sismos produjeron en la infraestructura de servicios. Trataremos de demostrar que, no obstante la destrucción y el evidente desgaste que representa la reconstrucción, los sismos abrieron nuevas oportunidades para la reforma y literalmente allanaron el camino para su profundización. Habremos entonces de analizar también el grado al que esta coyuntura ha sido favorable para los propósitos del actual régimen, examinando ios obstáculos y problemas que se le han presentado.

El contexto social y político

\section{La reforma institucional de la salud}

A lo largo de su proceso de desarrollo económico y social, México ha experimentado cambios importantes en las dos esferas centrales que definen el ámbito de la salud: por un lado, las condiciones de la población, esto es, el perfil de salud, enfermedad y muerte; por el otro lado, la respuesta que la sociedad organiza, a través de los servicios de salud, para hacer frente, con grados variables de efectividad, a las condiciones de la población.

Al lado de sus transiciones económica y demográfica, México atraviesa actualmente por una compleja transición epidemiológica. Ésta se

\footnotetext{
* Centro de Investigaciones en Salud Pública, SSA.

* * Dirección General de Epidemiología, SSA.
} 
caracteriza por un aumento en la incidencia de enfermedades crónicodegenerativas, padecimientos mentales y accidentes, al propio tiempo que persisten niveles altos de infecciones y desnutrición (Omran, 1971; González Block y Frenk, 1986).

No debe suponerse, como se hace a menudo, que el auge de las enfermedades cardiovasculares y el cáncer es un signo de progreso sanitario. Por el contrario, la transición no resuelta que vive México parece reflejar los efectos de un proceso de urbanización e industrialización que ha asignado baja prioridad al bienestar individual y colectivo. Más aún, la crisis económica ha dado pie a una especie de "contratransición", por la cual varias enfermedades que habían sido controladas en el pasado empiezan a resurgir, como es eı caso del paludismo y el dengue.

Hacia finales de la década de los años setenta empezó a formarse un consenso nacional e internacional en el sentido de que el modelo dominante de organización de la atención a la salud resultaba inadecuado para hacer frente a las complejas realidades de países como México. Este modelo se había caracterizado, entre otros, por los siguientes elementos: fragmentación del esfuerzo público en salud a través de la coexistencia de múltiples instituciones descoordinadas; centralismo en la toma de decisiones y en la operación de los servicios; asignación desproporcionada de recursos a los hospitales, con una debilidad consecuente de los centros de salud y otras unidades de primer nivel; predominio de especialistas poco capacitados para enfrentar íntegramente los problemas del individuo, la familia y la comunidad, y escasa participación de la población en la solución de sus problemas de salud.

El gobierno que inició su mandato a finales de 1982 decidió alterar de manera fundamental el modelo dominante de atención a la salud, a través de una profunda reforma de este ámbito de la política social. Paradójicamente, la crisis económica sirvió para dar un lugar prioritario a la salud dentro de los proyectos del nuevo régimen. En el pasado, una política común ante las crisis económicas había sido el recorte de los programas sociales. En 1982 la profundidad de la crisis permitió descubrir a la salud y a otros campos de la política social como espacios de acción indispensables para compensar los peores efectos del desajuste económico y evitar un deterioro drástico de los niveles de vida de las mayorías. En esta forma, la salud se convirtió en uno de los ejes del proyecto gubernamental por alcanzar una sociedad más igualitaria.

La visibilidad política ha dado factibilidad organizativa a la reforma de la atención a la salud. Ello se ha traducido en una serie de esfuerzos sin precedentes por proteger el gasto público en salud. Por supuesto, la presión de la deuda externa ha llevado a que se destine una mayor proporción del gasto gubernamental a su amortización, con lo cual el destino relativo del total del gasto al sector salud se ha visto decrecido. No obstante, descontando el pago de la deuda pública, es claro que el porcentaje 
del gasto federal destinado a salud fue en aumento (Secretaría de Salud, 1985), de $6.3 \%$ en 1983 a $8.2 \%$ en 1985 . Esto contrasta con el descenso que observó de 1978 a 1982 , de 10.1 a 6.4 por ciento. A pesar de la concordancia entre el aumento relativo al gasto en salud y las intenciones políticas, en términos reales el apoyo federal se ha visto menguado por la inflación.

Con todo, el hecho de que la salud haya sido una de las áreas del gasto público menos afectadas por los recortes presupuestales ha hecho posible llevar a la práctica la reforma sanitaria. Uno de los primeros pasos de esta reforma consiste en introducir una enmienda constitucional que establece el derecho de todo individuo a la protección de la salud. Una de las premisas de esta enmienda es que la libertad, la igualdad y la justicia son nociones vacías a menos que todos los mexicanos hayan alcanzado un nivel básico de vida. Según esta concepción, la atención a la salud no es una mercancía, un privilegio o un objeto de caridad, sino que se convierte en un derecho social para todos los ciudadanos.

Una enmienda formal a la Constitución es sólo el primer paso en un proceso prolongado. Evidentemente, el principio de asignación de servicios de salud con base en la ciudadanía implica una obligación para el Estado. Por esta razón, en febrero de 1984, el congreso aprobó una nueva Ley General de Salud, que interpreta el mandato constitucional en términos específicos. La ley define una serie de servicios de salud básicos; el gobierno asume la responsabilidad de su acceso universal. Más adelante, en agosto de 1984, el Programa Nacional de Salud fue aprobado como un instrumento de política que especifica las medidas requeridas para lograr el objetivo deseado de un sistema nacional de salud con cobertura universal.

Estas innovaciones legales y programáticas han proporcionado el marco para una reforma de las bases organizacionales de la salud en México, expresadas en cinco estrategias primordiales: descentralización, sectorización, modernización administrativa, coordinación sectorial y participación comunitaria. Con base en estas estrategias se enfrentarían los problemas de ineficiencia administrativa, falta de adecuación de los servicios a los problemas de salud de la población, desperdicio de recursos locales, y el verticalismo y autoritarismo característicos del modelo de desarrollo ya caduco.

Daños a la salud producidos por los sismos

\section{Salud física}

El total de muertes certificadas legalmente a causa de los sismos ascendió a cerca de 5000 (Sepúlveda, 1986), aunque el número de personas desa- 
parecidas podría incrementar la cantidad de víctimas. No hay du da de que la cifra es alta en términos absolutos. Sin embargo, debe hacerse notar que, en relación con la magnitud del temblor y con la densidad de la población en riesgo, éstos han sido probablemente de los sismos que menos muertes han causado.

Según las fuentes (Grupo de Prospectiva, 1986; G. Soberón, J. Frenk y J. Sepúlveda, 1986), se calcula que aproximadamente entre 10000 y 40000 personas fueron atendidas por todas las instituciones de salud durante los primeros cinco días después del primer sismo. Según datos del Departamento del Distrito Federal, del total de los atendidos $94 \%$ recibieron atención de consulta externa mientras que 6\% (2 348 personas) fueron hospitalizadas; de éstas $8.6 \%$ fueron intervenidas quirúrgicamente. Del total de hospitalizados se reportó que $1.7 \%$ fallecieron dentro de los servicios de hospitalización; $3.75 \%$ de los hospitalizados requirieron de prótesis y rehabilitación física. Los daños más frecuentes en los pacientes hospitalizados fueron los traumatismos, la insuficiencia renal aguda y la neurosis postraumática. En $54 \%$ de los heridos las lesiones fueron causadas por aplastamientos; en $38 \%$ la lesión se debió a caídas y en $8 \%$ a atrapamientos $\mathrm{u}$ atropellamientos.

La distribución de la población atendida según su género fue aproximadamente de 1:1; $27 \%$ tenía menos de 15 o más de 65 años de edad, lo cual indica que la población infantil resultó menos afectada, si se toma en consideración su representación nacional de más de $50 \%$.

Más de 900 de las muertes ocurrieron dentro de unidades hospitalarias, afectando a pacientes, enfermeras, médicos y personal paramédico, lo cual representa casi $20 \%$ de las muertes certificadas y alrededor de $10 \%$ del total de muertes probables (Cisero Sabido et al., 1986: 523).

\section{Salud mentaI}

La respuesta por parte de los profesionales de la salud mental ante el desastre fue muy amplia y variada, lo cual amerita un estudio en sí mismo. Casi 2000 psiquiatras, psicólogos y trabajadores sociales participaron en el auxilio de más de 12700 personas ubicadas en viviendas provisionales y campamentos. Se atendió adicionalmente a 5700 personas en otros lugares, así como a 2500 rescatistas. También funcionó un servicio telefónico de asesoría, el cual atendió más de 4500 llamadas (Tovar Acosta, 1985).

El Instituto Mexicano del Seguro Social (IMSS) puso en práctica un programa para la superación de crisis psicológicas dirigido a los damnificados, así como a quienes presentaban crisis psicopatológicas agudas. El programa se puso en práctica en albergues, fábricas y el espacio comunitario en general. La mayor parte de los beneficiarios fueron derechohabientes de esta institución. El programa de atención preventiva y terapéu- 
tica se llevó a cabo por medio de la participación comunitaria, se capacitó a cerca de mil promotores de salud y a cien supervisores sobre las respuestas colectivas más comunes frente a los desastres, las características psicológicas del duelo individual y las técnicas de intervención en crisis (Pucheu, 1986).

El programa de atención psicológica del IMSS se vio acompañado de un esfuerzo de investigación sobre las características epidemiológicas del daño. Se detectó que la población cubierta presentaba en $73 \%$ de los casos crisis leves y compensadas. De esta población, $16 \%$ sufrió crisis que presentaba descompensación moderada, mientras que $11 \%$ presentaba descompensación aguda (Padilla y cois., 1986).

El Instituto Mexicano de Psiquiatría y la Dirección General de Epidemiología llevaron a cabo una investigación conjunta sobre las características de los daños a la salud mental en la población de alto riesgo constituida por los damnificados en los albergues. Por medio del estudio se determinó que el síndrome de stress postraumático se presentó en $16 \%$ de esta población, resultados que coinciden en buena medida con los encontrados por los investigadores del IMSS.

El DIF llevó a cabo un programa de atención a las necesidades psicológicas de los damnificados que llegaron a sus albergues. Dicho programa fue apoyado por un equipo interdisciplinario del Instituto Nacional de Salud Mental (Martínez Zarandona, 1986). Por otra parte, diversas dependencias de la Universidad Nacional Autónoma de México (UNAM) prestaron ayuda psicológica. La Facultad de Psicología puso en práctica un servicio telefónico con escaso éxito y elaboró un manual de atención psicológica a escolares (Grupo de Prospectiva; 1986: 43). La Facultad de Medicina y la Dirección de Servicios Médicos prestaron ayuda psicológica a la población de alto riesgo.

Por su parte, la Asociación Mexicana de Psicoterapia Analítica de Grupo (AMPAG) preparó a psicólogos profesionales y voluntarios en la asistencia en situaciones de crisis por medio de los seminarios de formación de emergencia (Carrillo, 1986). Sus objetivos fueron proporcionar los instrumentos teóricos, técnicos y operacionales para enfrentar las emergencias psicológicas, individuales y colectivas. Un grupo trabajó en colaboración con UNICEF en la preparación de maestros como agentes de salud pública para la prevención de la enfermedad mental en niños.

En el curso de sus actividades, los miembros de la AMPAG reflexionaron sobre el papel del psicólogo y su identidad ante situaciones de desastre. En esta forma se llegó a importantes conclusiones teóricas sobre la formación de asistentes, la efectividad de diversas formas de terapia analítica de grupo y las características de los problemas enfrentados (Socorro, 1986; Orozco, 1986; Althaus, Lenz y Orozco, 1986).

La Asociación Psicoanalítica Mexicana (APM) también participó en la ayuda psicológica en diversos niveles y de diferentes formas. Una tesis 
que orientó buena parte de su acción fue que los medios de comunicación masiva tuvieron el principal papel etiológico en el trauma psicológico observado después de los sismos (Palacios, Cueli, Camacho et al., 1986). La ruptura de la comunicación telefónica nacional llevó a una sobrecarga emocional de los canales de comunicación masivos, aunado a la magnitud ya de por sí desproporcionada del desastre. Se calcula que en horas pico, los sismos atrajeron la atención de más de 20 millones de teleespectadores, así como del total de la población nacional por radio. En esta forma, el trauma psicológico fue magnificado, más aún después del segundo sismo. Ante esta tesis algunos miembros de la APM se abocaron principalmente a la atención de la población joven, considerada como la más afectada. En un programa especial dirigido a niños, se abarcaron 48 escuelas (Cuevas y Noriega, 1986) con terapia grupal. La APM también trabajó en la capacitación de multiplicadores de asistencia psicológica (Dupont, 1986), en la asistencia directa a damnificados en los albergues (Dallal y Castillo, 1986; Cueli, 1986), así como con casos especiales de damnificados que estuvieron atrapados en los escombros (Remus Araico, 1986).

\section{Factores condicionantes de la salud}

A pesar de que las mayores áreas de vivienda no fueron afectadas, 60000 personas perdieron su hogar. Un mes después de los temblores había todavía 29000 personas viviendo en casas provisionales y en campamentos. El número de gente que quedó sin servicio de agua potable fue al principio de más de 6 millones. No obstante, un mes más tarde esta cifra se había reducido a 90000 . A pesar de ello no se reportaron brotes de epidemias relacionadas con la carencia de líquido. En las primeras horas después de los sismos se montó una operación de vigilancia para controlar la calidad del agua, dar vacunación antitetánica así como para disponer de los cadáveres y evitar brotes epidémicos en los albergues (Sepúlveda, 1986).

\section{Daños a los servicios de salud}

La parte de la ciudad más severamente afectada abarcó una importante concentración de hospitales. El cuadro 1 muestra que las pérdidas absolutas y relativas de infraestructura fueron muy altas, considerándose como el peor desastre de servicios médicos en la historia de México. Los daños ocurrieron casi exclusivamente en unidades hospitalarias generales y de especialidades, quedando prácticamente intacta la red de atención ambulatoria.

El Centro Médico Nacional del IMSS, principal centro hospitalario del país y de América Latina, tuvo que ser completamente evacuado, ya que 


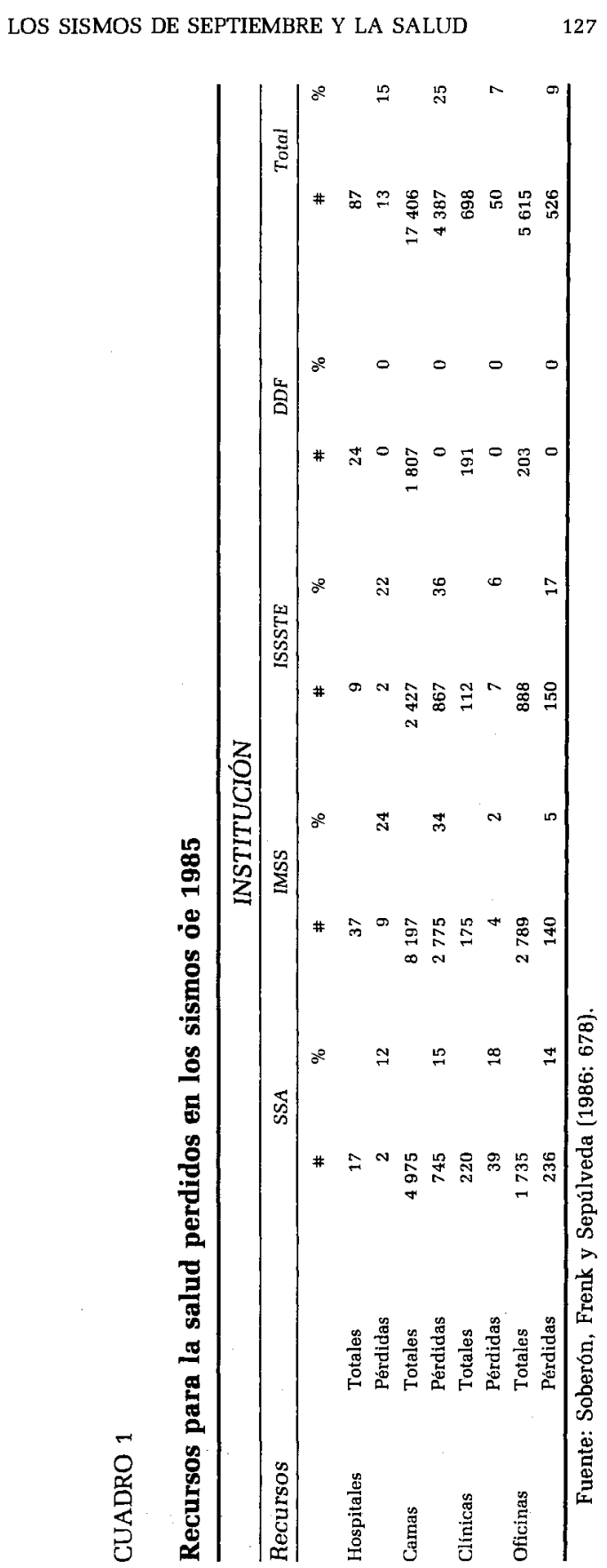


casi todos sus veinticinco edificios sufrieron daños. Cerca de 3000 pacientes tuvieron que ser evacuados, mientras que 22000 trabajadores fueron reubicados. La mayoría de sus 2306 camas estaban dedicadas a la atención de alta especialidad y representaban una tercera parte del total de las camas del IMSS en el área metropolitana. Por su parte el Instituto de Seguridad Social al Servicio de los trabajadores del Estado (ISSSTE), perdió 867 camas, las cuales representaban $36 \%$ de su capacidad (Valdés, 1986: 12-14).

La institución que mayores pérdidas sufrió en proporción a su capacidad instalada fue la propia Secretaría de Salud, la cual vio fuera de servicio de manera inmediata 2158 camas, $43 \%$ del total en el área metropolitana. Más de 700 de estas camas se perdieron definitivamente con el derrumbe del Hospital Juárez y la torre de ginecobstetricia del Hospital General de México, donde también se cayó la residencia de médicos. En comparación, la red de 24 hospitales generales con 1600 camas, perteneciente al Departamento del Distrito Federal (DDF) no fue afectada.

En total, la ciudad sufrió la pérdida en un solo día de más de 4000 camas del sector público, a las cuales deben agregárseles una menor cantidad perdidas por el sector privado. Cinco hospitales privados tuvieron que ser evacuados y decenas de consultorios privados se perdieron. Analizando el daño según las especialidades, no cabe duda de que la atención ginecobstétrica sufrió un daño desproporcionado; $41 \%$ de las camas hospitalarias del sector público en la zona metropolitana (tanto de instituciones de seguridad social como del gobierno federal) dedicadas a este tipo de atención quedaron inutilizadas, así como $32 \%$ de las salas de expulsión y de quirófanos de esta especialidad (González Block y Loewe, 1985).

Pese a las graves pérdidas materiales, no hubo escasez de camas para atender a los más de 2000 pacientes hospitalizados a raíz del primer temblor. Ello se debió tanto a la buena coordinación que se dio entre los hospitales de los diversos sectores - todos los cuales atendieron a población sin distinciones de capacidad económica o derechos-y a que se dieron de alta a pacientes hospitalizados para cirugía electiva. No obstante, esta suficiencia habla también de una abundancia de camas en la zona metropolitana, las cuales no estaban siendo adecuadamente utilizadas.

Los daños sufridos por los hospitales a causa de los sismos llevaron a una redistribución de las camas a disposición de la población no asegurada del Distrito Federal. Puede afirmarse que los sismos llevaron a la reducción de la concentración de camas en ciertas delegaciones, abatiendo paradójicamente la desigualdad imperante hasta la fecha (Grupo de Prospectiva, 1986: 56). Para que este efecto fuera permanente, sería necesario que la reconstrucción repusiera las camas perdidas en las delegaciones y estados que mostraban una afluencia de pacientes hacia el D.F. debida a la falta de recursos más accesibles.

Hasta antes del desastre, la delegación Cuauhtémoc contaba con una 
cama por cada 104 habitantes, lo cual la colocaba como la delegación con más disponibilidad de recursos para la atención hospitalaria. Esta situación contrastaba con delegaciones como Álvaro Obregón e Iztapalapa, sin ninguna cama hospitalaria para la población no asegurada, o Tláhuac y Contreras, con 6305 y 5568 habitantes por cama respectivamente.

Después de los sismos, la delegación Cuauhtémoc quedó con sólo 208 camas de las 2366 que tenía, lo cual la llevó a un índice de 1175 habitantes por cama. Este índice está muy cerca de las normas recomendadas por los organismos internacionales, lo cual permite ver la factibilidad de reconstruir las camas perdidas fuera de esta zona; reconstruir dentro llevaría de nuevo a incrementar la desigualdad en la distribución de las camas. Mientras que antes de los sismos el coeficiente de Gini para la distribución de las camas era de 0.23 , éste se vio reducido a 0.15 a raíz del desastre, lo cual es una muestra cuantitativa de la mayor igualdad distributiva.

\section{Afectación de la docencia}

La pérdida de hospitales a raíz de los sismos tuvo efectos que rebasaron la prestación de servicios para afectar a la formación de nuevas generaciones de médicos generales y especialistas. Se calcula que, como consecuencia inmediata de los sismos, se vieron afectados más de 3000 estudiantes de medicina, alumnos de los ciclos clínicos que recibían entrenamiento en el Hospital General y que provenian de las universidades Nacional Autónoma de México, Autónoma Metropolitana y La Salle, así como del Instituto Politécnico Nacional, cerca de 900 alumnos que asistían al Hospital Juárez, más 1100 que recibían enseñanza en el Centro Médico Nacional del IMSS, o sea, alrededor de 5000 estudiantes de los ciclos clínicos vieron interrumpida su enseñanza. Asimismo 650 internos de pregrado que concurrían a los hospitales General y Juárez y más de 300 residentes, se vieron afectados en el entrenamiento que recibían en esos centros (Martuscelli, Frenk, Ruelas y González Block, 1986).

\section{Oportunidades de la reconstrucción}

\section{Planes y prioridades}

La Secretaría de Salud tomó a su cargo la coordinación del Programa de Reconstrucción y Reordenamiento de los Servicios de Salud, basado en el principio rector de reponer la infraestructura perdida y a la vez reordenar los servicios para mejorar su operación (Valdés y Martínez Narváez, 1986; Coordinación Técnica para la Reconstrucción, 1986). Dicho programa retomó los planteamientos y sugerencias de los grupos de trabajo de la Coordinación de Salud del Comité de Auxilio Social, integrante de la Comisión Nacional de Reconstrucción (Comisión Nacional de Reconstrucción, 
1986). El Programa tiene caracter sectorial, ya que comprende las necesidades globales de la Secretaria de Salud, del DDF, del IMSS y del ISSSTE.

Entre las metas del Programa de Reconstrucción figuran las de abatir el déficit acumulado y lograr un mejor balance entre los tres niveles de atención médica; asegurar la atención de la demanda mediante el uso eficiente de la infraestructura remanente y la generación de capacidad hospitalaria adicional, a través de acciones de habilitación temporal y la construcción de nuevas unidades; favorecer el acceso de los usuarios, a través de una oferta más racional en las distintas regiones del país, especialmente en la zona metropolitana y en los estados aledaños al D.F.

Para poner en práctica el Programa de Reconstrucción y Reordenamiento quedó instalada la Coordinación Técnica de Reconstrucción de la Infraestructura Hospitalaria en la Zona Metropolitana de la Ciudad de México. Por otra parte se estableció una unidad ejecutora del Programa dentro de la Oficialía Mayor de la SSA (Valdés y Martínez Narváez, 1986).

\section{Consideraciones financieras}

El Programa de Reconstrucción y Reordenamiento de los Servicios de Salud está siendo financiado por diversas instancias, que son: el Fondo Nacional de Reconstrucción, un crédito especial del Banco Interamericano de Desarollo, el presupuesto ordinario de las dependencias del sector, donativos específicos no canalizados a través del Fondo de Reconstrucción, apoyos a cargo de la partida para reconstrucción que figuran en el presupuesto de egresos de la federación y por utilidades de la Lotería Nacional para la Asistencia Pública (Valdés, 1986: 40-41).

El costo total del Programa de Reconstrucción asciende a 148385 millones de pesos, de los cuales se espera sean financiados por el Fondo Nacional de Reconstrucción 31526 millones. El costo total de la reconstrucción para la población no asegurada asciende a 56808 , o sea, $38.3 \%$ del total. Casi una tercera parte de esta suma será financiada por el Fondo Nacional de Reconstrucción. La Secretaría de Salud es la principal institución en este esfuerzo de reconstrucción, con 37377 millones de pesos (Valdés, 1986: 42-44).

Las instituciones de seguridad social, principalmente por medio del IMSS, absorberán 91577 millones de pesos en su reconstrucción, de los cuales 12847 serán aportados por el Fondo Nacional de Reconstrucción. El programa del IMSS tiene un costo total de 62502 millones de pesos; de éstos, 8286 se aplicarán a tareas de rehabilitación y 54216 millones a la reconstrucción de centros médicos en los estados, de seis hospitales de segundo nivel y del Centro Médico Siglo XXI.

La reconstrucción del ISSSTE tiene un costo de 29075 millones de pesos; de ellos, 25406 se destinan a la construcción de tres hospitales gene- 
rales, a la construcción de un hospital de alta especialidad y al reordenamiento regional del instituto. El resto se aplicará a la rehabilitación de clínicas de adscripción y hospitales generales (Valdés, 1986: 46).

\section{Prospectiva de los servicios de salud}

A la semana de haber ocurrido el siniestro se formó un grupo de prospectiva sobre los servicios de salud, en respuesta a los graves daños sufridos por este sector. El grupo fue constituido por diversas instituciones académicas y de servicio (Grupo de Prospectiva, 1986) ${ }^{*}$ quienes de manera interdisciplinaria abordaron el problema de establecer el impacto de los sismos sobre los servicios de salud, así como diferentes alternativas para enfrentar los daños sufridos por el sector. Orientado principalmente al subsector que atiende a la población no asegurada —el sector con mayores daños per cápita-, el grupo concluyó que, a pesar de las camas puestas inmediatamente fuera de servicio, este subsector en su conjunto cubriría:

. . la actual demanda, presentando una proporción de $2.86 \%$ en la relación hospitalización/consulta, con 2615 camas. Esto significa que, dado que se cuenta con 4418 camas, se tiene un superávit de 1803 para cubrir la actual demanda (Grupo de Prospectiva, 1986: 56)

Se llegó a esta conclusión bajo el supuesto de que el total de las camas de segundo nivel que resistieron el sismo estarían a disposición solamente de la población no asegurada del Distrito Federal, que ascendía a 2754 millones de pesos para 1985. Ello supone a su vez que la reconstrucción estaría orientada a la distribución de camas en la zona conurbada del Estado de México, por lo cual las camas en el D.F. quedarían en su mayor parte a disposición de los capitalinos. La conclusión a la que se llegó sustenta estos supuestos, ya que, visto desde la perspectiva de la población, no era necesario reedificar ninguna cama en el D.F.

Los cálculos anteriores se apoyan en la tasa de consulta/hospitalización encontrada hasta antes de los sismos. Se sabe, no obstante, que la población no asegurada carece de la suficiente atención de consultas y, por lo tanto, de una atención idónea a su salud. El Grupo de Prospectiva planteó la posibilidad de que la población recibiera cuatro consultas por persona por año, las cuales llevarían a reducir la tasa de hospitalización, en la

* De la UNAM participaron el Programa Universitario de Investigación Clínica, el Instituto de Investigación en Matemáticas Aplicadas y Sistemas, el Centro Universitario de Tecnología Educacional para la Salud, el Centro de Información Científica y Humanística, la Facultad de Medicina, la Escuela de Bibliotecologia y la Dirección General de Asuntos del Personal Académico. Por parte de la Secretaría de Salud participaron el Centro de Investigaciones en Salud Pública y el Instituto Mexicano de Psiquiatría. Participó también la Dirección General de Servicios Médicos, del DDF. 
medida en que se diera una atención preventiva más eficaz. Se planteó como un supuesto que el índice de consulta/hospitalización podría reducirse en un tercio debido a este factor. Bajo esta serie de supuestos se concluyó que:

En conjunto la SSA y los SMDDF requieren de 4004 camas para cubrir la demanda deseable de 600 hospitalizaciones diarias. Dado que se cuenta con 4418 camas, se tendría un superávit de 414 camas (Grupo de Prospectiva, 1986: 55).

Si no existía una necesidad inmediata de reedificar - ni siquiera de reabrir el Hospital General-, ¿cuál era la urgencia de reedificar camas en el Distrito Federal? El Grupo de Prospectiva consideró las proyecciones de población no asegurada y su demanda de camas hospitalarias a dos años con el fin de contestar a esta pregunta. Las conclusiones fueron:

La proyección de demanda deseable con reducción en la proporción hospitalización/consulta a dos años es la siguiente: los Servicios Médicos del DDF muestran un déficit de 1532 camas mientras la Secretaría de Salud tiene un superávit de 1252 . Globalmente se tiene un déficit de 280 camas. En lo referente a consultorios. . . de manera global el superávit es de 416 consultorios. . . De no operar una reducción de un tercio en la proporción hospitalización/consulta se tiene a dos años un déficit. . . global de 2627 camas (Grupo de Prospectiva, 1986: $62)$.

La investigación prospectiva deja ver la necesidad del reforzamiento del primer nivel de atención, esto es, de la atención no hospitalaria, con vistas a la prevención de la enfermedad en general y como forma de disminuir la demanda por camas hospitalarias. Los sismos presentaron entonces no sólo un reto para mejorar la distribución de las facilidades de segundo nivel, sino también para modificar la organización de los servicios y la accesibilidad que éstos guardan respecto a la población no asegurada.

Quedó establecido entonces que la reedificación de hospitales en el Distrito Federal era una medida a todas luces injustificada, no sólo por el problema del riesgo sísmico y la alta densidad urbana sino, sobre todo, por la nula necesidad actual y escasa necesidad a mediano plazo. Con esta perspectiva puede analizarse de manera más objetiva el proceso que llevó a la reapertura casi total del Hospital General. La disputa por la reapertura inmediata de este nosocomio puso en evidencia una de las limitaciones de la reforma iniciada con este sexenio, donde se buscaba dar prioridad a la atención primaria descentralizada (Frenk, 1986).

Esta limitación estriba en la reticencia de los grupos médicos en modificar su forma de organización, centrada en los grandes hospitales que por 
lo común están alejados de las necesidades más inmediatas de la población. Si bien el proyecto de la reforma fue aceptado ante la perspectiva de que no tocaría los grandes enclaves médicos, los sismos llevaron la reforma literalmente hasta el quirófano y obligaron a los médicos a una súbita e inesperada defensa de sus intereses. Ante la necesidad de contener cualquier movilización por parte de los grupos médicos, el gobierno se vio en la necesidad de ceder ante sus demandas, máxime que éstas fueron planteadas bajo el estandarte populista. En efecto, el cierre del Hospital General fue exhibido ante la opinión pública como una afrenta contra una institución social de larga trayectoria, verdadero baluarte de la justicia y asistencia popular. No cabe duda de que el Hospital General fue esto y más en su momento. Lo que no hay que olvidar, sin embargo, es que el tiempo suele cambiar el contexto de las instituciones para modificar su significado y tornarlas en trabas al desarrollo, si no logran adaptarse a los nuevos tiempos.

\section{Acciones emprendidas}

Se ha señalado que las estrategias de reconstrucción comprenden cuatro opciones, las cuales no son mutuamente excluyentes: la reedificación, la rehabilitación, la redistribución y la reorganización (Soberón, Frenk y Sepúlveda, 1986).

\section{Reedificación y rehabilitación}

La reedificación se limitó a la recuperación de las instalaciones que quedaron dañadas, pero cuyas estructuras eran recuperables. Ningún hospital fue reconstruido tal y como estaba antes si su estructura había quedado completamente dañada. Aun en el caso del Centro Médico Nacional, la obra en proceso representa una reorganización, ya que se construirán hospitales de una dimensión mucho menor a la original, recuperándose el resto de las camas en proyectos en la periferia de la ciudad.

No obstante lo limitado de la reedificación, ésta tuvo de cualquier forma una dimensión considerable. El Hospital General fue reabierto en su totalidad, con excepción de la unidad de ginecobstetricia y la residencia médica. Del Hospital Juárez será recuperado el viejo casco así como el edificio de investigación, los cuales no quedaron destruidos. Por otra parte, se construirá una nueva torre de hospitalización de tercer nivel en una zona diferente de la ciudad. No obstante, en este caso debe hablarse de una reorganización, ya que el Hospital Juárez asumirá nuevas funciones. Otros siete hospitales de segundo nivel, siete institutos nacionales y 26 centros de salud de primer nivel fueron reparados. En total se erogó 
la cantidad de 4207 millones de pesos, equivalente a $11.26 \%$ de los 37377 millones de pesos destinados a la Secretaría de Salud dentro del Programa de Reconstrucción.

\section{Redistribución}

La redistribución de camas hospitalarias a raíz de los sismos fue considerable. Ya se habló de la redistribución espontánea, así como de la factibilidad de aprovechar el desastre para lograr una accesibilidad hospitalaria más equilibrada. De acuerdo con el Programa de Reconstrucción (Ortega Lomelín, 1986) se destinarán 16179 millones de pesos a la construcción de seis hospitales de 144 camas de segundo nivel en la zona conurbada de la ciudad de México. Los hospitales están siendo construidos en los municipios de Atizapán de Zaragoza, Ecatepec, Cuautitlán Izcalli, Toluca, Naucalpan y Nezahualcóyotl, todos ellos en el Estado de México. En su conjunto cubrirán a una población de 1.5 millones de habitantes. Este esfuerzo representa un cambio sin precedentes en el sistema de salud metropolitano, ya que revierte la altísima concentración hospitalaria que existía en la delegación Cuauhtémoc.

La redistribución con base en los nuevos hospitales pretende ser un primer paso para una reorganización más amplia, basada en una concepción de un sistema de servicios de salud, hasta ahora incipiente. Con este fin, todo el personal directivo de los nuevos hospitales fue sujeto a cursos intensivos en el país y en el extranjero para capacitarlos en la organización y dirección ya no sólo de los hospitales, sino de un sistema de atención médica integrad $\rho$ por diferentes niveles que responda a las necesidades de una población dentro de una región bién delimitada.

El IMSS está llevando a cabo también un importante esfuerzo de redistribución a raíz de los sismos, aunque este organismo ya tenía una trayectoria anterior que facilitó la consecución de sus planes. El planteamiento de reconstrucción comprende trasladar 838 camas a cinco ciudades del interior de la república; recuperar 1180 camas de segundo nivel con la adaptación de seis hospitales generales de zona en el valle de México, así como construir el Centro Médico Siglo XXI con sólo 600 camas en el mismo sitio donde antes funcionaban 2600 .

\section{Reorganización}

La reorganización de los servicios de salud es quizás el aspecto más interesante a que dio lugar el desastre de septiembre de 1985. Si bien existió, como se vio antes, una fuerte resistencia a los planes iniciales de reconstrucción, también se lograron grandes avances en la reforma gracias a los 
sismos septembrinos. Si la reapertura del Hospital General marca el caso más claro de reedificación, y la construcción de los hospitales conurbados borda entre la redistribución y la reorganización, los eventos que se evidencian en torno al Hospital Juárez marcan el más claro ejemplo de reorganización en los servicios de salud. En efecto, la casi total destrucción de esta antigua institución llevó a un replanteamiento al cual los intereses médicos tradicionales han opuesto muy poca resistencia. Por el contrario, los trágicos eventos llevaron a una visión más abierta del futuro de ese hospital.

En primer término debe plantearse la inminente construcción de un nuevo centro hospitalario de alta especialidad con un costo de 13865 millones de pesos, equivalentes a $37 \%$ del total de los fondos del Programa de Reconstrucción de la SSA. Este nuevo centro se ubicará en el norte del Distrito Federal, alejándose —no sin cierta resistencia- de la vieja área de influencia que el hospital tenía en el centro de la capital, pero logrando una distribución más equilibrada de los recursos para la atención médica de alta especialidad. Este nuevo hospital no marca en sí mismo la reorganización a la que nos referimos. Lo novedoso radica en la remodelación del viejo casco del hospital y del edificio de investigación, únicos edificios que resistieron el temblor. En ellos se instala actualmente un Centro Avanzado de Atención Primaria a la Salud (CAAPS), ideado a partir de los sismos como una respuesta a la devastación hospitalaria a la vez que a las urgentes necesidades de servicios de una metrópoli como la ciudad de México. Los CAAPS fueron diseñados por el Centro de Investigaciones en Salud Pública como un modelo general susceptible de reforzar el primer nivel de atención y disminuir los ingresos hospitalarios, respuesta comprobada en cuanto a su eficiencia teórica en la investigación del Grupo de Prospectiva a que aludimos antes.

En su concepción general, los CAAPS consisten en unidades no hospitalarias en las que se realizan funciones usualmente llevadas a cabo en los hospitales, aunque sin una verdadera justificación en una urbe como México. Tales funciones son la atención del parto normal, la cirugía ambulatoria, la consulta de especialidades y la atención general a poblaciones bien delimitadas con el fin de lograr la prevención y la cobertura de la población de alto riesgo. Estas funciones se realizarían bajo una novedosa organización basada en la práctica médica grupal, la delimitación de poblaciones específicas, un esquema de referencia a unidades hospitalarias y, de ser factible, una forma de acceso basada en el pago anticipado de los servicios. Con estas medidas se garantizaría una mayor cobertura poblacional así como calidad y eficiencia de los servicios. 
Aspectos de la ejecución

La Secretaría de Salud resolvió reorganizar los servicios del Hospital Juárez y del Hospital General con base en la propuesta de los CAAPS, así como también poner en práctica este modelo en centros de salud estratégicamente localizados en la ciudad. Para el Hospital Juárez la propuesta era muy atractiva, ya que le permitiría reutilizar el remanente de sus viejas instalaciones. Para el Hospital General la propuesta era atractiva porque le permitiría recuperar su capacidad de atención de partos, la cual no fue recuperada totalmente con la reedificación. Para otros centros de salud la propuesta era atractiva porque permitiría una mayor capacidad resolutiva, descongestionando así a los hospitales a la vez que disminuyendo el grave desperdicio de recursos que significa la subutilización de la capacidad instalada.

\section{El CAAPS del Hospital Juárez}

La innovación que representa el CAAPS en el Hospital Juárez no radica tanto en la idea original, sino en lo que de ella resultó en el contexto de un hospital tradicional de segundo y tercer nivel. El CAAPS vinculará al hospital, por medio de la medicina general familiar, con una población concreta del primer cuadro, dentro de la cual destacan los campamentos de damnificados. Por otra parte, y en un radio de cobertura más amplia, se captará a población proveniente de centros de salud para la cirugía ambulatoria y la consulta de especialidades, funciones que siempre fueron fuertes en el Hospital Juárez y que ahora se realizarán en el contexto de un nuevo esquema de referencia entre los centros de salud y el hospital.

Es de notar que el acomodo de un hospital con énfasis en las altas especialidades al modelo de atención primaria no fue fácil. Algunos médicos optaron por no participar de la innovación, o por participar tímidamente a través de la "rotación", yendo al CAAPS periódicamente desde sus hospitales de base. Aun cuando esta forma de vinculación tiene sus virtudes, el arreglo responde sobre todo a la poca comprensión del enfoque de la atención primaria, definiéndosele como medicina de primer nivel, si no es que primitiva. La atención primaria no puede ser definida de ninguna de estas dos maneras; es una forma de organizar servicios de todos los niveles con respecto a la población. El objetivo es que se proporcione una medicina integral - preventiva, curativa y básica a la vez que especializada-, a una población bién definida según sus necesidades de salud. No obstante, debe comprenderse que en México la medicina preventiva ha sido siempre opacada por los grandes hospitales centrados en la enfermedad y la alta tecnología. Esta no deja de ser la excepción ahora, con la perspectiva de la construcción del nuevo Hospital Juárez. Para con- 
trarrestar esta tendencia, se está buscando el mejor equipamiento del CAAPS, de tal forma que la consulta de especialidades y la cirugía ambulatoria tengan una alta calidad -igual o mejor a aquella practicada en los hospitales-, dándole así prestigio a esta nueva modalidad de organización.

En lo que toca a la práctica médica grupal y al acceso por medio del pago anticipado, estas innovaciones habrán de ser objetc de estudio dentro de un proyecto experimental. En efecto, una parte integral del modelo CAAPS es la realización de investigaciones sobre su eficiencia, costo y calidad. Los resultados permitirán extender el concepto a otras unidades de salud, así como ampliar la idea para incluir nuevas funciones. Sobre estas bases, el CAAPS se convierte en un modelo flexible y cambiante según las circunstancias y resultados, permitiendo así el surgimiento de la innovación que más se ajuste a las necesidades y organización de la metrópoli.

\section{Conclusiones}

Uno de los sectores de la vida social más afectados por los sismos de septiembre de 1985 fue el de la salud: las profesiones de la salud fueron las que sufrieron mayor número de muertos en el trabajo; la pérdida de instalaciones y equipo cuenta entre las más cuantiosas en la historia nacional y mundial, y la población se vio directa o indirectamente muy afectada por las pérdidas de este sector.

La magnitud del desastre define las dimensiones del reto de la reconstrucción. Desde los primeros días después de los sismos empezó a surgir un amplio consenso social en torno al principio de que la reconstrucción no podía simplemente restaurar las cosas a su estado previo. En efecto, los sismos mostraron la vulnerabilidad de un modelo de desarrollo basado en la concentración de recursos, que en el caso de la salud se vuelve una concentración doble: geográfica, al ubicar la mayor cantidad de recursos en una pequeña área de la capital, y tecnológica, al centralizar los elementos de la atención en los hospitales.

La vulnerabilidad de este modelo de desarrollo ha generado una responsabilidad por lograr que la reconstrucción constituya una auténtica renovación, capaz de sumarse a la reforma que el país ya venía llevando a cabo antes de los sismos. Después de décadas de predominio del modelo basado en la concentración urbana y hospitalaria de la atención, la reforma sanitaria había finalmente dado la más alta prioridad a la descentralización y a la atención primaria. Pero los sismos afectaron justamente a la contraparte, esto es, a la ciudad capital y a la atención de segundo y tercer nivel. Ello planteó el riesgo de que la reconstrucción volviera a canalizar recursos a estos ámbitos, desprotegiendo, de nueva cuenta, a los sectores tradicionalmente descuidados por las instituciones de Salud en México: la provincia y el primer nivel de atención. 
Si bien algunos de los movimientos políticos a que hemos aludido hicieron tangible la realidad de dicho riesgo, también es cierto que los sismos abrieron una excepcional oportunidad para profundizar la reforma, que hubiera sido impensable sin una catástrofe natural. Hemos mostrado que los sismos dieron lugar, por sí mismos, a una distribución menos desigual de recursos para la atención médica. Este proceso se ha visto reforzado por la decisión de enfocar el esfuerzo reconstructor en sitios de la zona metropolitana y del país que hasta ahora han padecido de una debilidad en su infraestructura de atención a la salud.

Los sismos también revelaron una importante capacidad de respuesta por parte de la comunidad académica. En efecto, se han producido investigaciones multidisciplinarias de alta calidad sobre distintos aspectos del desastre. Sería deseable que esta experiencia diera lugar a instancias concretas de coordinación de la investigación sobre problemas relacionados con desastres y emergencia.

En suma, el alto costo humano y económico de los sismos ha dado lugar, como contrapartida, a nuevas oportunidades de renovación. Aprovecharlos cabalmente seré la única forma de consolidar el cambio estructural en la salud para así dar sentido a la tragedia.

\section{Bibliografía}

Althaus Esther, Irene Lenz y Beatriz Orozco (1986), Los grupos ante la crisis, México, AMPAG, inédito.

Carrillo, José A. (1986), Lo que el sismo develó, México, AMPAG, inédito.

Cicero Sabido, Raúl, Antonio Padua, Héctor Rodríguez Martínez et al. (1986), "Efectos del terremoto del 19 de septiembre de 1985 en el Hospital General de la ciudad de México. Algunas consideraciones", en Salud Pública de México, 28 (1986), 521-526.

Coordinación Técnica para la Reconstrucción (1986), Programa de reconstrucción y reordenamiento de los servicios de salud, México, ssa.

Cueli, José (1986), La respuesta humana ante el desastre, México, APM, inédito.

Cuevas, Pablo e Ignacio Noriega (1986), Efectos del desastre civil en la ciudad de México, intervención psicológica, asesoría y participación en escuelas, México, APM, inédito.

Dallal y Castillo, Eduardo (1986), La ayuda psicológica de emergencia en desastres: el trabajo con damnificados de albergues, México, APM, inédito.

Dupont Muñoz, Antonio (1986), Capacitación de voluntarios como promotores de salud mental en situaciones de crisis, mediante técnicas grupales, México, APM inédito.

Frenk, Julio (1985), 'Las oportunidades de la reconstrucción", en Nexos, diciembre de 1985, 53-57.

González Block, M.A. y Ricardo Loewe, "Lo perdido y lo que ya no debe apareCer", en Nexos, 85, 1985, 57-59.

González Block, M.A. y Julio Frenk (1986), "El horizonte de la salud" en México mañana, México, Ediciones Océano-Nexos, pp. 85-95.

Grupo de Prospectiva (1986), Prospectiva de los servicios de salud a partir de los 
sismos de septiembre de 1985, México, Coordinación de la Investigación Científica, UNAM. (Cuadernes del PUIC, 2.)

López Cervantes, Malaquías. Lizbeth López Carrillo y José Antonio Escamilla Cejudo (1986), "Algunas consecuencias de los sismos de septiembre de 1985 en la ciudad de México", en Salud Pública de México, 28 (1986), 527-536.

Martínez Zarandona, Irene (1986), Programa de prevención y minimización de daños a población afectada por el desastre, México, DIF, inédito.

Martuscelli, J., J. Frenk, E. Ruelas y M.A. González Block (1986), “La formación de recursos humanos para la salud en el contexto de la reordenación y reconstrucción de los servicios en el Valle de México", en Revista de Educación Superior, 59 (1986), 5-14.

Omran, A. (1971), "The Epidemiologic Transition. A Theory of the Epidemiology of Population Change', en Millbank Memorial Fund Quarterly, 49 (1971), 509-538.

Orozco, Beatriz (1986), Los grupos como instrumento para abordar situaciones de crisis, México, AMPAG, inédito.

Ortega Lomelín, Roberto (1986), "El Programa Nacional de Salud y su financiamiento", en Le Monde Diplomatique en español, Suplemento, mayo de 1986.

Padilla, Paula y cols. (1986), Frecuencia de trastornos psicológicos en sectores de la población afectada por los sismos. Análisis de indicadores obtenidos en el IMSS, México, IMSS, inédito.

Palacios, Agustín, José Cueli, José Camacho et al. (1986), The Traumatic Effect of Massive Communication in the Mexico City Earthquake. Crisis Intervention and Preventive Mensures, México, APM, inédito.

Pucheu, Carlos (1986), Desastre sísmico y crisis psicológicas. Modelo de intervención preventiva desarrollado por el IMSS, México, IMSS, inédito.

Remus Araico, José (1986), Reflexiones psicosociales sobre salud mental en la investigación de casos de atrapamiento del terremoto de septiembre, México, APM, inédito.

Secretaría de Salud (1985), Impacto de la crisis económica en la salud, México, Dirección General de Estadística e Informática.

Sepúlveda, Jaime (1986), "Vigilancia epidemiológica posterior a los sismos de septiembre de 1985", en Boletín Mensual de Epidemiología, 1986, núm. 1, 4-7.

Soberón, Guillermo, Julio Frenk y Jaime Sepúlveda (1986), "The Health Care Reform in Mexico: Before and After the 1985 Earthquakes", en American Journal of Public Health, 76 (1986), 673-680.

Socorro, Héctor (1986), Los terremotos: una identidad para el psicólogo, México, AMPAG, inédito.

Tovar Acosta, Héctor (coord.) (1985), Programa de Salud Mental relacionado con el desastre: informe de acciones desarrolladas (25 de septiembre a 15 de octubre), México, SSA, inédito.

Valdés, Cuauhtémoc (1986), Reconstrucción y reordenamiento de los servicios de salud, una visión panorámica, México, Secretaría de Salud, inédito.

Valdés, Cuauhtémoc y Gregorio Martínez Narváez (1986), "Reconstrucción y reordenamiento de los servicios de salud", en Le Monde Diplomatique en español, suplemento, mayo de 1986. 
